\title{
Effects of Acute Low Back Pain on Postural Control
}

\author{
Min Kyun Sohn, MD, Sang Sook Lee, MD, Hyun Tak Song, MD
}

Department of Rehabilitation Medicine, Chungnam National University School of Medicine, Daejeon, Korea

\begin{abstract}
Objective To evaluate the changes in static and dynamic postural control after the development of acute low back pain.

Methods Thirty healthy right-handed volunteers were divided into three groups; the right back pain group, the left back pain group, and the control group. $0.5 \mathrm{~mL}$ of $5 \%$ hypertonic saline was injected into L4-5 paraspinal muscle for 5 seconds to cause muscle pain. The movement of the center of gravity (COG) during their static and dynamic postural control was measured with their eyes open and with their eyes closed before and 2 minutes after the injection.

Results The COGs for the healthy adults shifted to the right quadrant and the posterior quadrant during their static and dynamic postural control test $(\mathrm{p}<0.05)$. The static and dynamic instability index while they had their eyes closed was significantly increased than when they had their eyes open with and without acute back pain. After pain induction, their overall and anterior/posterior instability was increased in both the right back pain group and the left back pain group during the static postural control test $(\mathrm{p}<0.05)$. A right deviation and a posterior deviation of the COG still remained, and the posterior deviation was greater in the right back pain group $(\mathrm{p}<0.05)$. Conclusion The static instability, particularly the anterior/posterior instability was increased in the presence of acute low back pain, regardless of the visual information and the location of pain.
\end{abstract}

Keywords Low back pain, Postural control, Balance

\section{INTRODUCTION}

Functional balance is an essential element in one's gait and daily life activities involving sight, hearing, vestibular

Received April 10, 2012; Accepted August 28, 2012

Corresponding author: Hyun Tak Song

Department of Rehabilitation Medicine, Chungnam National University Hospital, Chungnam National University School of Medicine, 282 Munhwa-ro, Jung-gu, Daejeon 301-721, Korea

Tel: +82-42-280-7811, Fax: +82-42-256-6056, E-mail: smfahen@cnuh. co.kr

(c) This is an open-access article distributed under the terms of the Creative Commons Attribution Non-Commercial License (http://creativecommons. org/licenses/by-nc/3.0) which permits unrestricted noncommercial use, distribution, and reproduction in any medium, provided the original work is properly cited.

Copyright $\odot 2013$ by Korean Academy of Rehabilitation Medicine apparatus, proprioceptive sense, position sense, muscular force, and cognition [1]. Postural control is achieved by the integration of the information regarding body movement sensed through the somatosensory system in the central nervous system and the appropriate reaction of the musculoskeletal system [2]. Proprioception is sensed from mechanoreceptors of muscles, ligaments, and joints and retains stability and bearings of the body during its static and dynamic movements $[3,4]$.

Mok et al. [5] has found that an efficiency of postural recovery after an unexpected perturbation becomes lowered in people with chronic back pain and it is associated with poor use of spinal motion as a component of the postural strategy. Nies and Sinnott [6] found that 
low back pain patients had greater postural sway and were less likely to keep their balance on one foot with their eyes closed, compared to healthy people. Moreover, Mientjes and Frank [7] reported that a significant increase in medial/lateral instability in chronic low back pain patients was found during performance of tasks involving the removal of vision, especially in combination with increased task complexity. It was hypothesized that the increased body sway in patients with low back pain results from, among other things, injury and/or damage to proprioceptive tissues in the lumbar spine. Also, abnormal electromyography (EMG) activities may be attributable to the impairment in postural control $[8,9]$.

However, previous studies about postural control were limited to the chronic back pain group, and had little evidence related with acute back pain. In several studies involving patients with acute back pain, delayed action time and reduced muscular activities of the trunk muscle were reported during their postural perturbation [10-12]. On the other hand, in patients with chronic back pain, changes of muscular activities were found not only in the trunk, but also in the lower extremities $[13,14]$. Thus, the postural control strategy in patients with acute back pain might be different from that in patients with a chronic back pain condition.

Based on this background, the aim of this study was to evaluate the changes of static and dynamic postural stability in patients with acute back pain. We also attempted to find differences in the instability pattern and the center of gravity (COG) distribution, depending upon the location of pain.

\section{MATERIALS AND METHODS}

\section{Subjects}

This study was conducted on 30 healthy right-handed adult subjects (16 males, 14 females) with a mean age of $24.7 \pm 2.0$ years. Individuals currently suffering from back pain, having a history of a lumbar and lower extremity operation, or a balance disorder were excluded from this study. A written consent for participation in this study was obtained from subjects prior to their participation, and then, they were grouped by random drawings in the order of their acceptance into the study; the right back pain group, the left back pain group, and the control group.

\section{Methods}

\section{Back pain induction}

To induce experimental low back pain in subjects, 0.5 $\mathrm{mL}$ of $5 \%$ hypertonic saline was slowly injected over 5 seconds into their L4-5 paraspinal muscles $5 \mathrm{~cm}$ lateral from the spinous process in a prone position. Subjects were asked to rate their pain on a scale from 0 to 10 , wherein 0 stood for no pain and 10 for the most severe pain imaginable. Maximal pain was induced between 3 to 5 minutes after injection and the maximal visual analogue scale (VAS) score was $4.15 \pm 1.22$. In the control group, normal saline was similarly injected to the subjects, and they complained of a mild pain (VAS score, $1.3 \pm 0.7$ ) only at the time when the needle was inserted.

\section{Evaluation of postural control}

Static and dynamic postural control was evaluated by using the Biodex Balance System SD (Biodex Medical System, Shirley, NY, USA) with eyes open and with eyes closed. Cachupe et al. [15] reported that as a result of investigating 20 subjects, the Biodex Balance System showed a reliable stability index. A static balance test was conducted on the subject standing on a fixed circular foot plate and aligning his or her COG to the center of the circular plate as shown on the monitor. Dynamic balance involved free tilting of the circular foot plate depending upon weight shifting. Therefore, when the subject's COG was deviated to one side, the foot plate began to tilt to the same side. If the degrees of free tilting were too high, there might be an increased risk of fall, so we set the static force to stage 6 of the 12 stages (Fig. 1).

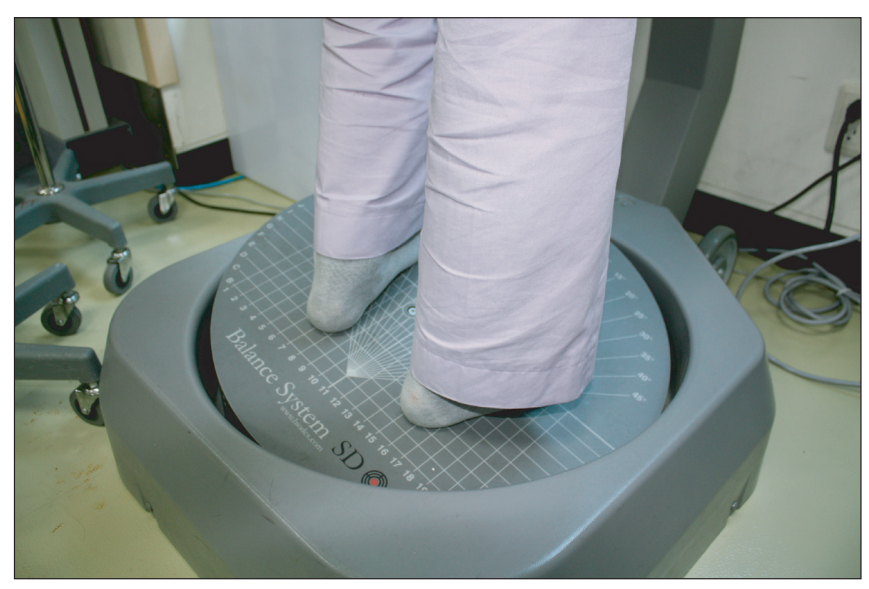

Fig. 1. Dynamic postural stability test. When subject's center of gravity was deviated to one side, the foot plate began to tilt to the same side. 
Body weight shifts were traced in a green line on the model plate, which was divided into 4 quadrants (anterior, posterior, left, and right), and the time durations spent in each zone were indicated in a percentage. Each distance for the overall, anterior/posterior, and medial/ lateral movement of the body weight shift was also measured. The results were recorded as the index score of overall instability (OAI), anterior/posterior instability (API), and medial/lateral instability (MLI), respectively. A high index score meant greater instability, and the OAI score was determined depending upon API and MLI. If a subject held the handle due to his/her loss of balance, 0.2 points per session was deducted from the OAI (Fig. 2).

Evaluations were carried out 2 minutes after injection into the paraspinal muscle. To minimize errors from adaptation, 2 exercise sessions were allowed beforehand. Each test consisted of 2 sessions lasting 20 seconds each and a 10 second break between sessions. Evaluations were classified into static and dynamic measures with eyes open at first and then with eyes closed. Before evaluations with the eyes closed, the subjects were asked to set their COG to the center of the circular plate before closing their eyes.

\section{Statistical analysis}

A statistical analysis was performed by SPSS ver. 12.0 (SPSS Inc., Chicago, IL, USA). The quadrant distribution before the injection at each condition and the COG deviation were analyzed by an independent t-test. The changes of the instability index between the condition of eyes open and the condition of eyes closed were verified by the paired t-test in each group. The OAI, API, and MLI were compared by the one-way analysis of variance (ANOVA) between group comparisons. Differences in quadrant deviation changes between the right back pain group, the left back pain group, and the control group were verified by the paired t-test. A mean and standard deviation was given to the values, and a p-value less than 0.05 demonstrated statistical significance.

\section{RESULTS}

\section{Postural control in the healthy condition}

During the static postural control test with eyes open and closed, the COGs of healthy right handed adults were shifted to the right quadrant compared with the left quadrant $(82.8 \pm 18.8 \%$ with eyes open, $80.9 \pm 25.9 \%$

Postural stability test results

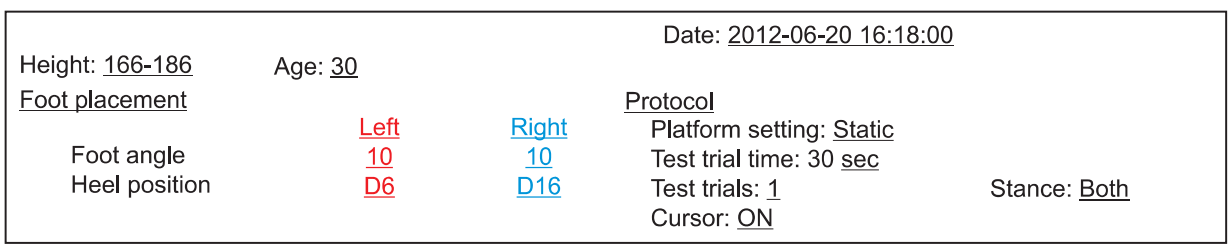

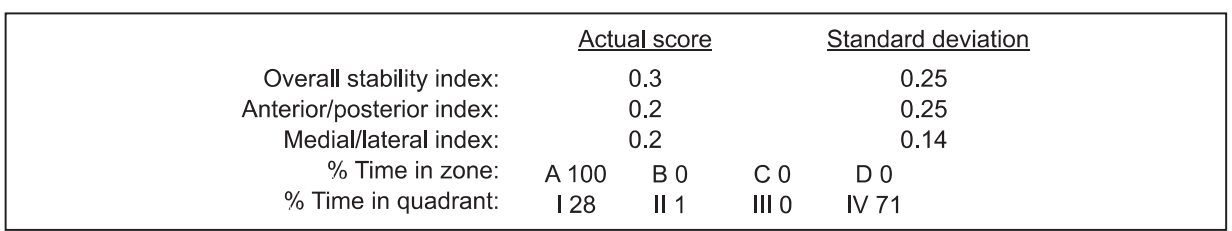

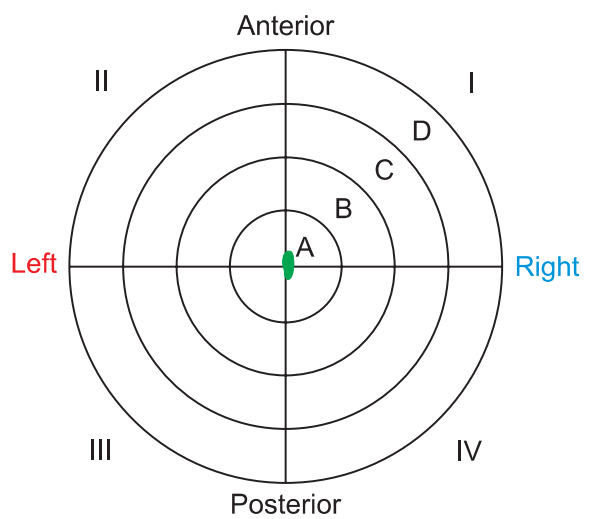

Fig. 2. The result sheet of the postural stability test. Movement from the center of gravity can be seen in the green trace line. Overall, anterior/posterior, medial/lateral instability indexes, and percentage of time in the quadrant were measured. 
with eyes closed) and the posterior quadrant compared with the anterior quadrant $(73.6 \pm 11.0 \%$ with eyes open, $72.8 \pm 17.6 \%$ with eyes closed), both with a statistical significance $(\mathrm{p}<0.05)$. During the dynamic postural control test with eyes open and closed, the COGs were also shifted to the right quadrant $(66.1 \pm 17.8 \%$ with eyes open, $57.2 \pm 15.6 \%$ with eyes closed) and the posterior quadrant ( $81.3 \pm 19.1 \%$ with eyes open, $63.2 \pm 19.3 \%$ with eyes closed), both with a statistical significance $(\mathrm{p}<0.05)$ (Fig. 3).

For the static postural instability index, the OAI, API, and MLI indexes were more significantly increased with eyes closed than with eyes open (OAI, $0.43 \pm 0.16$ to
$0.81 \pm 0.37$; API, $0.28 \pm 0.12$ to $0.62 \pm 0.33$; MLI, $0.23 \pm 0.13$ to $0.38 \pm 0.27$ ), and those were also significantly increased with eyes closed than with eyes open in the dynamic postural stability test (OAI, $0.7 \pm 0.28$ to $2.46 \pm 1.40$; API, $0.53 \pm 0.26$ to $1.74 \pm 0.90$; MLI, $0.34 \pm 0.17$ to $1.37 \pm 0.95$; p<0.05) (Fig. 4).

Postural instability in the presence of acute low back pain

The preinjection evaluation showed that the static and dynamic OAI, API, and MLI had no significant differences between groups. After the induction of pain, static
(A)

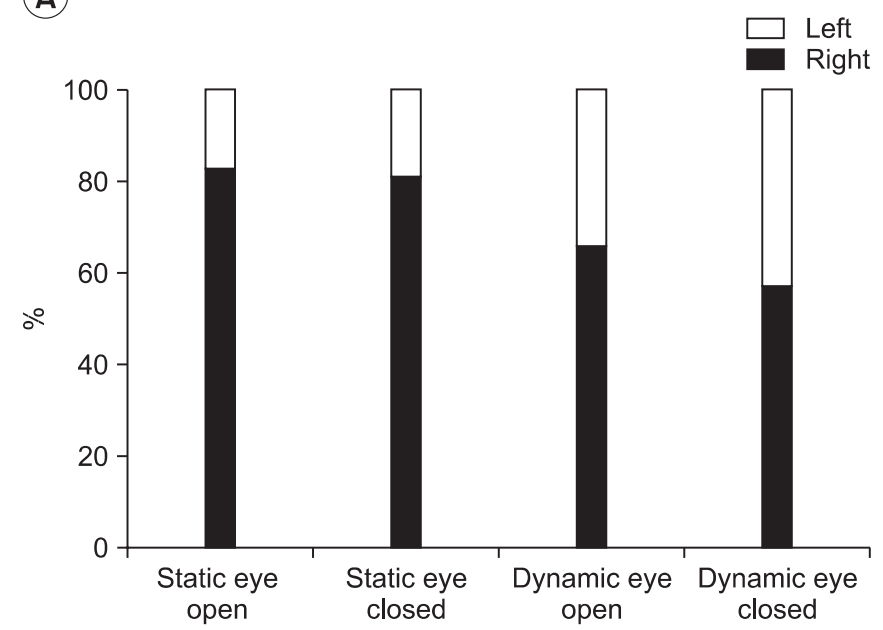

(B)

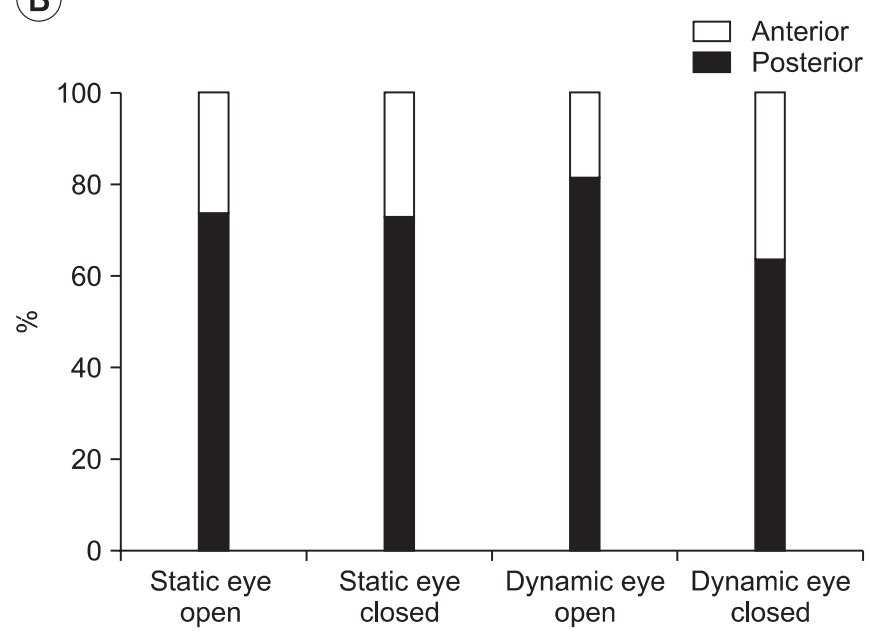

Fig. 3. The static and dynamic postural stability test showed that the proportion of the center of gravity was significantly deviated to the right side compared to the left side (A), and to the posterior side compared to the anterior side (B). $\mathrm{p}<0.05$ by independent $\mathrm{t}$-test.

(A)

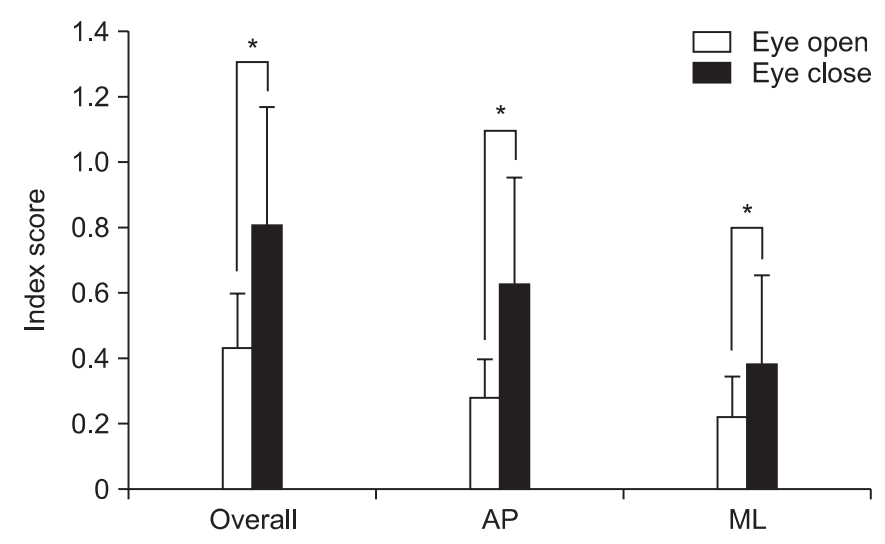

(B)

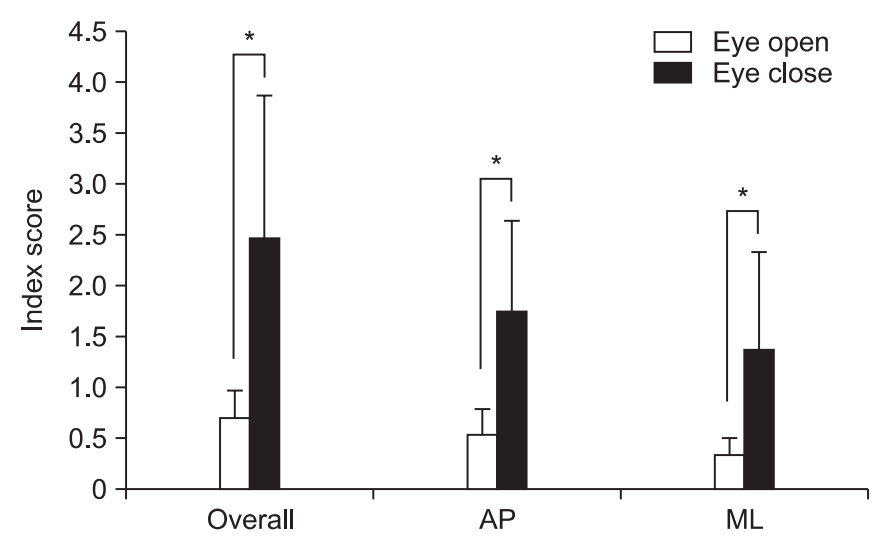

Fig. 4. The overall, anterior/posterior (AP), and medial/lateral (ML) instability indexes increased significantly with eyes closed than with eyes open during the static (A) and the dynamic postural stability test $(\mathrm{B}) .{ }^{*} \mathrm{p}<0.05$ by paired $\mathrm{t}$ test. 
(A)

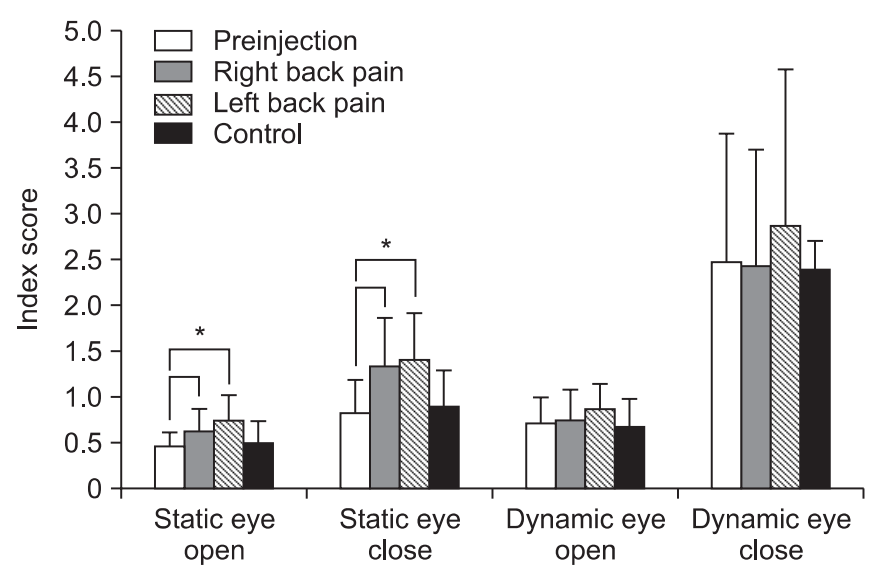

(C)

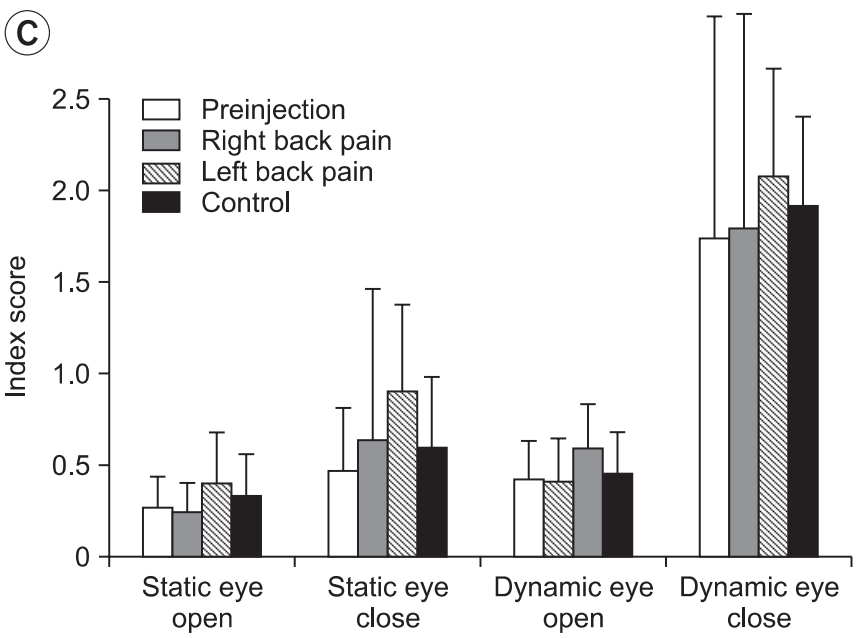

OAI and API increased significantly in both of the right back pain groups (OAI, $0.41 \pm 0.14$ to $0.61 \pm 0.24$ with eyes open and $0.72 \pm 0.43$ to $1.32 \pm 0.53$ with eyes closed; API, $0.26 \pm 0.10$ to $0.52 \pm 0.27$ with eyes open and $0.60 \pm 0.45$ to $1.07 \pm 0.31$ with eyes closed) and the left back pain groups (OAI, $0.42 \pm 0.19$ to $0.62 \pm 0.27$ with eyes open and $0.90 \pm 0.34$ to $1.38 \pm 0.52$ with eyes closed; API, $0.28 \pm 0.15$ to $0.42 \pm 0.21$ with eyes open and $0.65 \pm 0.25$ to $1.05 \pm 0.44$ with eyes closed) during the static postural control test $(\mathrm{p}<0.05)$. However, MLI showed no significant change after inducing pain. On the other hand, during the dynamic postural control test, no significant change was shown in any instability index after the induction of acute back pain (Fig. 5).

Changes of anterior/posterior and medial/lateral weight shift in the presence of acute low back pain

Even after inducing low back pain through an experimental procedure, there still existed right and posterior deviation. Interestingly, only posterior deviation in the
(B)

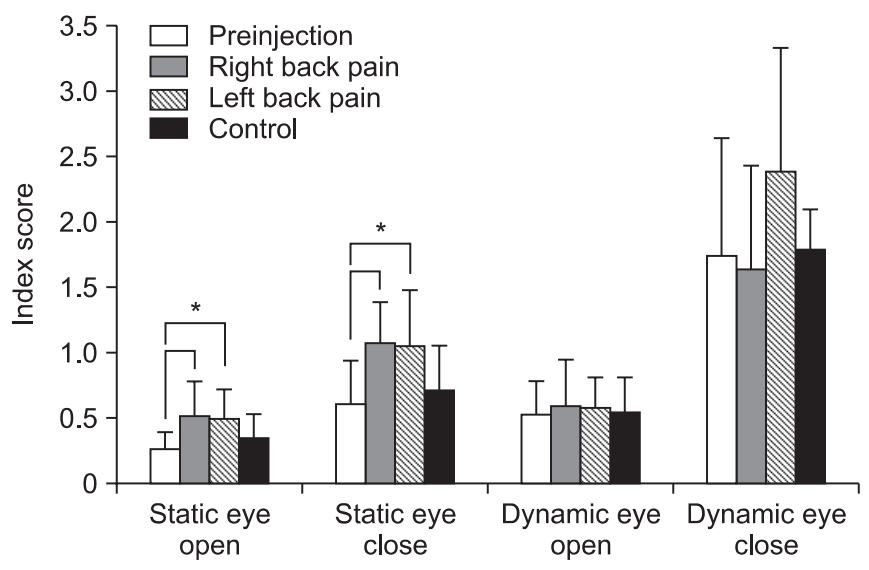

Fig. 5. Instability index after induction of the experimental low back pain increased significantly in the static postural stability test and in the overall instability (A), anterior/posterior instability test (B), and medial/lateral instability (C) compared to those of preinjection. ${ }^{*} p<0.05$.

right back pain group during the static postural control test was more significantly increased from $75.5 \pm 28.0 \%$ to $87.8 \pm 11.7 \%$ with eyes open and from $73.4 \pm 15.8 \%$ to $86.9 \pm 11.8 \%$ with eyes closed $(\mathrm{p}<0.05)$. No significant change was observed in anterior/posterior and medial/ lateral weight shift in the dynamic stability test (Fig. 6).

\section{DISCUSSION}

This study investigated a postural control pattern in a healthy population with eyes open and with eyes closed in the static state and the dynamic condition, with the influence of experimental acute back pain during postural control. The results revealed 1) a significant deviation in the right quadrant and the posterior quadrant for both the static and the dynamic postural control test in regards to both the healthy condition and the acute low back pain groups; 2) OAI, API, and MLI were more significantly increased while eyes were closed than while eyes 
(A)

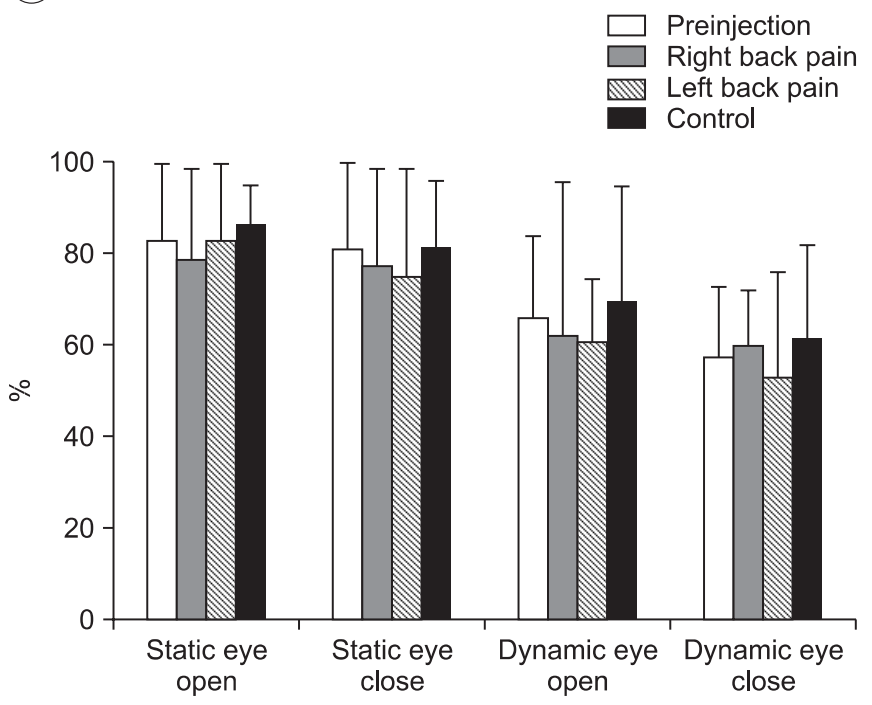

(B)

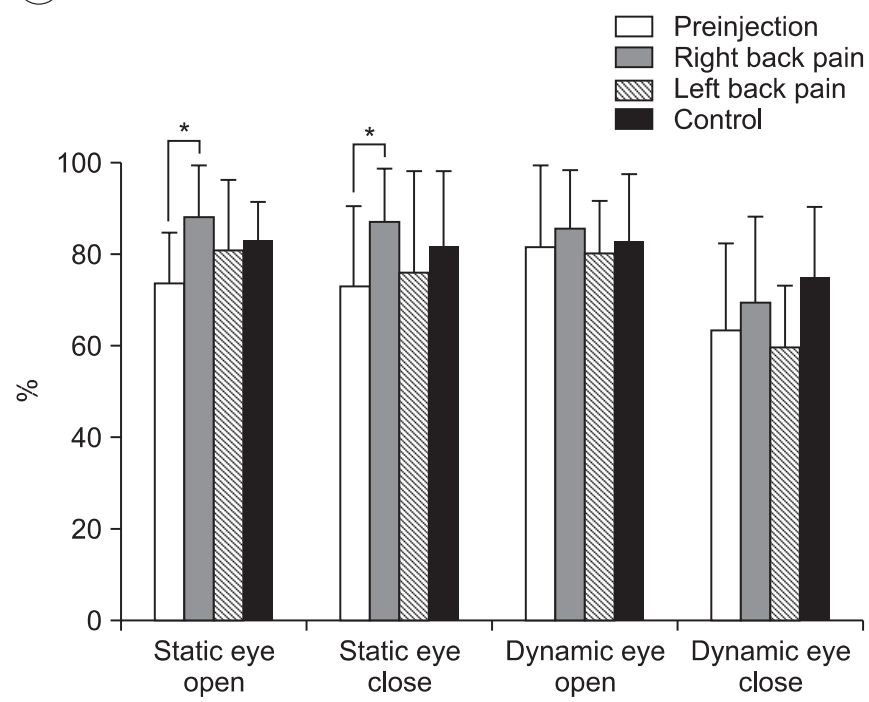

Fig. 6. The postural deviation toward the right side still existed even after induction of the experimental low back pain in the static postural control test (A). Interestingly, the right low back pain group (B) showed greater posterior side deviation after induction of the pain during the static postural stability test. ${ }^{*} \mathrm{p}<0.05$.

were opened during both the static postural control test and the dynamic postural control test in both the healthy condition and the acute low back pain; 3) OAI and API were both significantly increased during the static postural control test with no significant change during the dynamic postural control test after the pain induction; and 4) a greater posterior deviation of COG after induction of right back pain during the static postural control test.

While someone is standing quietly, his body makes continuous movements even with his feet fixed on the ground. Such movements are small and reflexive processes to maintain his postural equilibrium. Since a human being has a high center point of mass and a small support base, he has difficulty maintaining a vertical posture. However, the multi-joints structure of the body allows a man to keep his balance in a variety of body configurations, even while he is in motion. It is considered that one's ability to rapidly adjust the movement timing of a muscle in response to any unexpected postural perturbation is very important for maintaining posture and balance.

Alterations in somatosensation from the limbs or the trunk, however, have been shown to modulate automatic postural responses [16]. Postural control involves the coexistence of stability and mobility. Hip and spine articulations are thought to contribute to the maintenance of postural stability $[17,18]$. Activity began with the ankle joint muscle and then radiated in sequence to the thigh muscle and then the trunk muscle on the same dorsal or ventral side of the body [19].

Recent studies have estimated that $14 \%$ of the United States population or more suffer from pain related to joints and the musculoskeletal system. Muscular injuries are a common cause of disability in the population, and muscle problems are the most common cause of low back pain [20]. There were a lot of reports about postural instability and impaired balance control in patients with low back pain $[5,7,9,21]$. They reported that patients with low back pain had MLI in most cases and API in some cases while eyes were closed. These results were more significant in the aspect regarding increased task complexity. Some authors asserted that increased MLI resulted from decreased proprioception and decreased spinal movement while one's posture was maintained.

Individuals with chronic low back pain demonstrated delayed and reduced EMG activity in the trunk muscle in response to postural perturbations $[19,22]$. In other studies, patients with low back pain showed decreased gluteus medius activity, which could be associated with reduced hip stability [13]. Jacobs et al. [14] reported that subjects with chronic low back pain revealed less earlyphase activity at the internal oblique muscle and the gastrocnemius muscle and less late-phase activity at the 
erector spinae muscle, the rectus abdominis muscle, and the tibialis anterior muscle. Pandy et al. [23] reported that one's mediolateral balance during walking was maintained by the hip abductor, the vastus muscle groups, the soleus muscle, and the gastrocnemius muscle. Thus, abnormal muscular activity not only in the trunk muscle but also in muscles of the lower extremities in patients with low back pain was one of the reasons for their MLI.

Regarding the acute back pain, Nelson-Wong et al. [24] reported that prolonged standing, which induces back pain has some negative impact on the balance response. This study showed increased static postural instability, particularly in the anterior/posterior direction after the induction of experimental back pain. Thus, the postural stability strategy to maintain balance may be different between acute back pain and chronic back pain patients.

In patients with experimental acute back pain, Boudreau et al. [12] investigated the muscular activity in the erector spinae muscle and the external oblique muscle. This study showed a delay in the onset time and a decrease in the amplitude of EMG activity for both muscles in response to whole-body perturbation after the induction of pain. Hodges et al. [11] reported a constant reduction of the external oblique abdominal muscle activity after the experimental pain induction, compared with the erector spinae muscle. Thus, imbalanced and abnormal muscular activities of the flexor muscle and the extensor spinal muscle might be the reason for the API while pelvic and hip muscular functions were maintained in patients with acute back pain.

della Volpe et al. [25] reported that subjects with chronic low back pain showed an increased sway during the dynamic postural control test, and Radebold et al. [9] reported that patients with chronic low back pain demonstrated poorer balance performance at the higher level of task difficulty, which revealed similar results compared to our study. This difference might result from the changes in muscular activity in the chronic back pain group. Krause et al. [26] performed five types of weightbearing tests and reported that the activity of the hip abductor increased greatly while standing with one foot on the irregular surface, compared to standing with two feet on the flat surface. This result is supported by Clark et al. [27], who reported that in the dynamic torso extension exercise, the activity of the hip flexor and biceps femoris increased more greatly than that of the paraspinal muscle when the exercise intensity increased. It means that the lower extremity muscle played an important role in dynamic postural control. Interestingly, our study results showed that there was no significant change in instability during the dynamic postural control test after the induction of pain. We can assume that both trunk muscles and lower extremity muscles are used to maintain balance in the dynamic condition while mainly trunk muscles are used in the static condition, so there was little effect of injection in the paraspinal muscle during the dynamic postural control test. Another hypothesis is that although the changes of the instability index were not statistically significant, those also tended to increase after the induction of pain. Therefore, if the sample size had been much larger, it could have shown a significant change.

In our study, both the right deviation and the posterior deviation in the static postural control test were significant in the normal healthy population as well as in the acute back pain condition. The increase in the posterior deviation is thought to be related to the increase in API. In contrast to the study conducted by Lim et al. [28], where they evaluated the balance of chronic backache patients using the Balance Master system (NeuroCom, Clackamas, OR, USA) our study showed a trend of the patients deviating toward the painless side; the right back pain group and the left back pain group showed left deviation and right deviation, respectively. These findings are thought to be a habituated avoidance response for reducing pain caused by the paralumbar and pelvic muscular spasm while the patient is standing. Their results are different from those of our study, and the reason for the differences is unclear. Thus, further study is needed.

As discussed above, further investigation is required to find out whether muscular activities of the lower extremities would be altered, and whether the COG pattern differs from that in patients with chronic back pain. Also, further investigation is required on whether right handed subjects utilize the right spinal muscle more frequently while maintaining their balance.

As a result of measuring postural stability changes in subjects with experimental low back pain, the overall instability was increased regardless of the visual information and location of pain in the static postural control test. Particularly, the increase in API had a significant role. In the dynamic evaluation, the postural instability was not changed regardless of the visual information 
and the pain location as the pelvic and lower extremity muscles may be not affected.

\section{CONFLICT OF INTEREST}

No potential conflict of interest relevant to this article was reported.

\section{ACKNOWLEDGMENTS}

This study was financially supported by research fund of Chungnam National University in 2009.

\section{REFERENCES}

1. Prado JM, Stoffregen TA, Duarte M. Postural sway during dual tasks in young and elderly adults. Gerontology 2007;53:274-81.

2. Nashner LM. Sensory, neuromuscular, and biomechanical contributions to human balance. In: Duncan PW, editor. Balance: proceedings of the APTA Forum; 1989 Jun 13-15; Nashville, TN. Alexandria, VA: American Physical Therapy Association; 1990.

3. Newcomer KL, Laskowski ER, Yu B, Johnson JC, An $\mathrm{KN}$. Differences in repositioning error among patients with low back pain compared with control subjects. Spine (Phila Pa 1976) 2000;25:2488-93.

4. Laskowski ER, Newcomer-Aney K, Smith J. Proprioception. Phys Med Rehabil Clin N Am 2000;11:323-40.

5. Mok NW, Brauer SG, Hodges PW. Changes in lumbar movement in people with low back pain are related to compromised balance. Spine (Phila Pa 1976) 2011;36:E45-52.

6. Nies N, Sinnott PL. Variations in balance and body sway in middle-aged adults: subjects with healthy backs compared with subjects with low-back dysfunction. Spine (Phila Pa 1976) 1991;16:325-30.

7. Mientjes MI, Frank JS. Balance in chronic low back pain patients compared to healthy people under various conditions in upright standing. Clin Biomech (Bristol, Avon) 1999;14:710-6.

8. Hodges PW, Richardson CA. Delayed postural contraction of transverses abdominis in low back pain associated with movement of the lower limb. J Spinal Disord 1998;11:46-56.

9. Radebold A, Cholewicki J, Polzhofer GK, Greene HS.
Impaired postural control of the lumbar spine is associated with delayed muscle response times in patients with chronic idiopathic low back pain. Spine (Phila Pa 1976) 2001;26:724-30.

10.Zedka M, Prochazka A, Knight B, Gillard D, Gauthier $M$. Voluntary and reflex control of human back muscles during induced pain. J Physiol 1999;520 Pt 2:591604.

11. Hodges PW, Moseley GL, Gabrielsson A, Gandevia SC. Experimental muscle pain changes feedforward postural responses of the trunk muscles. Exp Brain Res 2003;151:262-71.

12. Boudreau S, Farina D, Kongstad L, Buus D, Redder J, Sverrisdottir E, et al. The relative timing of trunk muscle activation is retained in response to unanticipated postural-perturbations during acute low back pain. Exp Brain Res 2011;210:259-67.

13. Notzel D, Puta C, Wagner H, Anders C, Petrovich A, Gabriel HH. Altered hip muscle activation in patients with chronic non-specific low back pain. Schmerz 2011;25:199-206.

14. Jacobs JV, Henry SM, Jones SL, Hitt JR, Bunn JY. A history of low back pain associates with altered electromyographic activation patterns in response to perturbations of standing balance. J Neurophysiol 2011;106:2506-14.

15. Cachupe WJ, Shifflett B, Kahanov L, Wughalter EH. Reliability of Biodex Balance System Measures. Meas Phys Educ Exerc Sci 2001;5:97-108.

16. Inglis JT, Horak FB, Shupert CL, Jones-Rycewicz C. The importance of somatosensory information in triggering and scaling automatic postural responses in humans. Exp Brain Res 1994;101:159-64.

17. Kantor E, Poupard L, Le Bozec S, Bouisset S. Does body stability depend on postural chain mobility or stability area? Neurosci Lett 2001;308:128-32.

18. Hodges PW, Gurfinkel VS, Brumagne S, Smith TC, Cordo PC. Coexistence of stability and mobility in postural control: evidence from postural compensation for respiration. Exp Brain Res 2002;144:293-302.

19. Horak FB, Nashner LM. Central programming of postural movements: adaptation to altered supportsurface configurations. J Neurophysiol 1986;55:136981.

20. Magni G, Caldieron C, Rigatti-Luchini S, Merskey H. Chronic musculoskeletal pain and depressive symp- 
toms in the general population: an analysis of the 1st National Health and Nutrition Examination Survey data. Pain 1990;43:299-307.

21. Leinonen V, Kankaanpaa M, Luukkonen M, Kansanen M, Hanninen O, Airaksinen O, et al. Lumbar paraspinal muscle function, perception of lumbar position, and postural control in disc herniation-related back pain. Spine (Phila Pa 1976) 2003;28:842-8.

22. Sohn MK, Yoon YS, Jun KH. EMG power spectrum of lumbar back muscle in chronic low back pain patients. J Korean Acad Rehabil Med 1998;22:68-76.

23. Pandy MG, Lin YC, Kim HJ. Muscle coordination of mediolateral balance in normal walking. J Biomech 2010;43:2055-64.

24. Nelson-Wong E, Howarth SJ, Callaghan JP. Acute biomechanical responses to a prolonged standing exposure in a simulated occupational setting. Ergonomics
2010;53:1117-28.

25. della Volpe R, Popa T, Ginanneschi F, Spidalieri R, Mazzocchio R, Rossi A. Changes in coordination of postural control during dynamic stance in chronic low back pain patients. Gait Posture 2006;24:349-55.

26. Krause DA, Jacobs RS, Pilger KE, Sather BR, Sibunka SP, Hollman JH. Electromyographic analysis of the gluteus medius in five weight-bearing exercises. J Strength Cond Res 2009;23:2689-94.

27. Clark BC, Manini TM, Mayer JM, Ploutz-Snyder LL, Graves JE. Electromyographic activity of the lumbar and hip extensors during dynamic trunk extension exercise. Arch Phys Med Rehabil 2002;83:1547-52.

28. Lim KB, Lee HJ, Joo SJ, Lim SS. Postural control measures of patients with lower back pain using balance master system. J Korean Acad Rehabil Med 2007;31: 30-6. 